$$
\mathbf{p H}=\mathbf{8 . 3 2}
$$

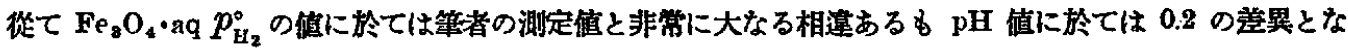
れり。

\title{
總括
}

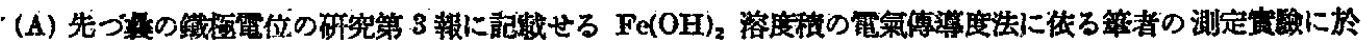

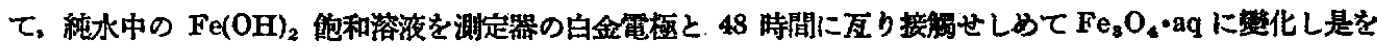

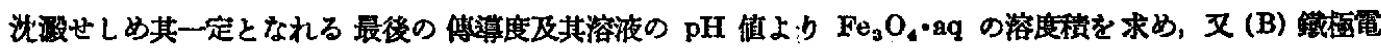

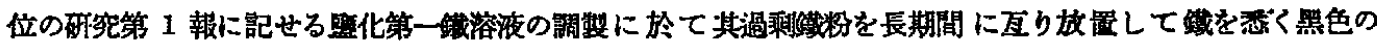

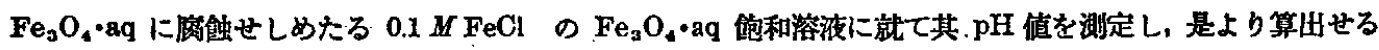

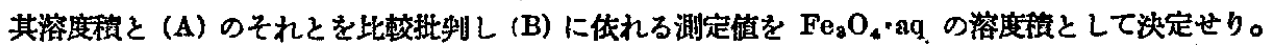

$$
\left[a_{\mathrm{Fe}}++\times a_{\mathrm{Fe}}^{2}+++a_{\mathrm{OB}}-\right]^{\circ} \mathrm{Fes}_{4} \cdot 2 \mathrm{qq}=10^{-103}[\mathrm{~mol} l l]
$$

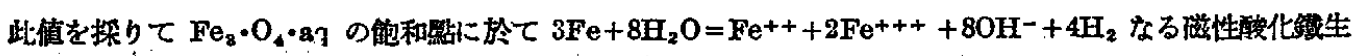

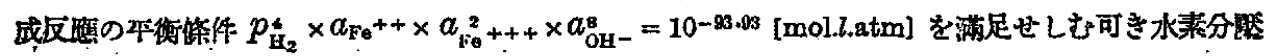

$$
\mathrm{Fe}_{3} \mathrm{O}_{4} \cdot \mathrm{ag} \boldsymbol{P}_{\mathrm{H}_{2}}^{\prime}=233 \mathrm{~atm}
$$

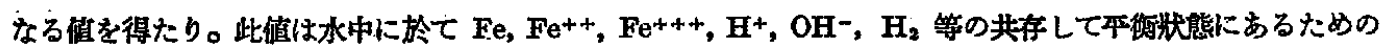

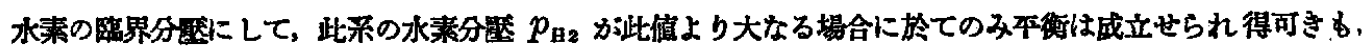

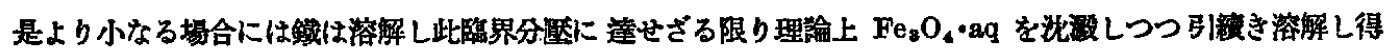
可き事を示す。

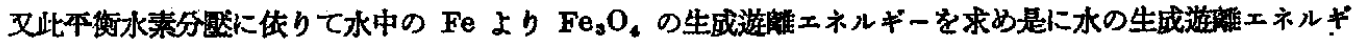
一を加算して $\mathrm{Fe}_{3} \mathrm{O}$ 、の組成元素よりの生成遊辟エネルギーを次の如く算定せり。

$$
3 \mathrm{Fe}(\mathrm{s})+2 \mathrm{O}_{2}=\mathrm{Fe}_{2} \mathrm{O}_{4} \text { (s; } \quad \Delta \mathrm{F}_{298}=-239065 \mathrm{cal}
$$

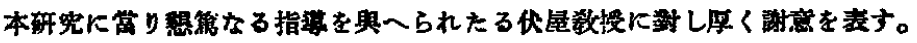

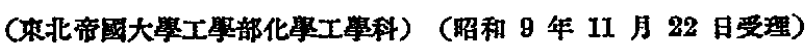

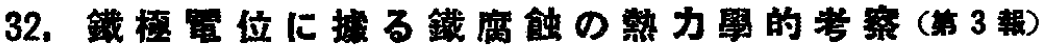 水素氣涯下に於ける鐵の腐蝕機柫}

\section{村田宽 夾}

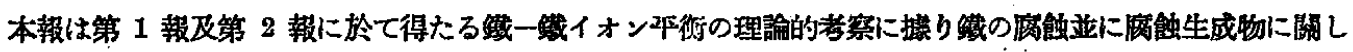

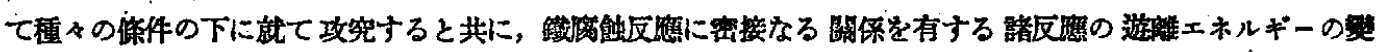

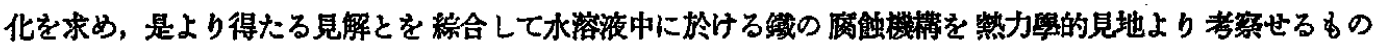
なり。

鐵の腐蝕並に腐蝕生成物に韮する和見

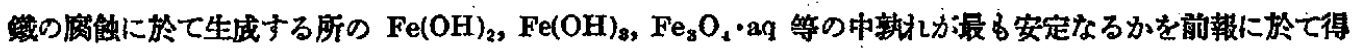
たる結果を綜合して種々の路件の下に就て吟味せんとす。

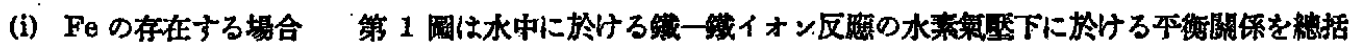


せるすのにして第 1 報 (8)，(18) 及 (23) なる平矢關俰式を圆示せるるのなり。

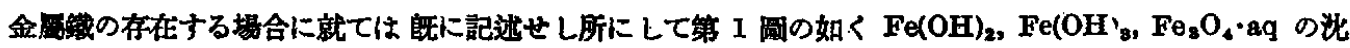

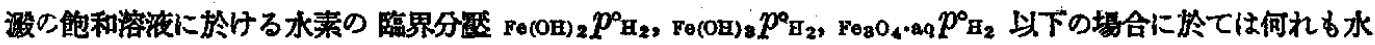

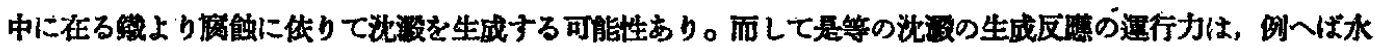

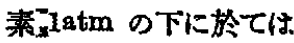

$$
\begin{aligned}
& E_{\mathrm{Fe}(\mathrm{OH})_{\mathrm{s}}}=\frac{R T}{2 F} \ln _{\mathrm{Fo}(\mathrm{OH})_{3}} p^{\circ}{ }_{\mathrm{H}_{2}}=0.02958 \log 10=0.0296 \mathrm{~V} \quad 25^{\circ} \mathrm{C} \\
& E_{\mathrm{Fe}(\mathrm{OH})_{2}}=\frac{R T}{2 F} \ln {\mathrm{Fe}(\mathrm{OH})_{2}}_{2} p_{\mathrm{H}_{2}}^{\circ}=0.03958 \log 12.5=0.0325 \mathrm{~V} \text { " }
\end{aligned}
$$

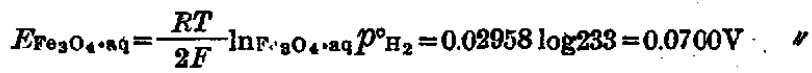

第 1 国

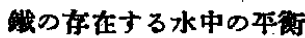

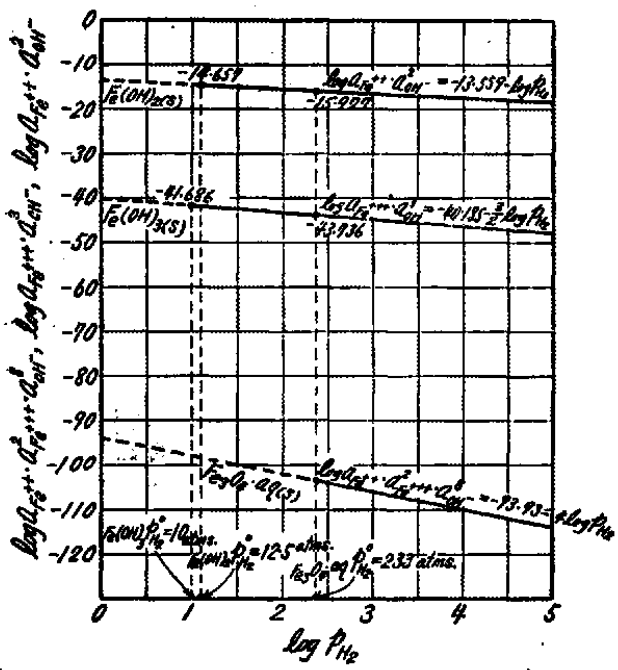

にして是等の生成反礁力何れる進行するすのとせば 本來 $\mathrm{Fe}(\mathrm{OH})_{2}$ 沈浱の生成は $\mathrm{Fe}(\mathrm{OH})_{2}$ より容易に して叉 $\mathrm{Fe}_{3} \mathrm{O}_{4} \cdot \mathrm{aq}$ は $\mathrm{Fe}(\mathrm{OH})_{2(\mathrm{~s})}$ 又は $\mathrm{Fe}(\mathrm{OH})_{3(\mathrm{~s})}$ の

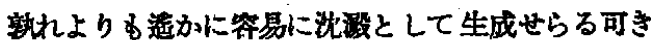
なり。

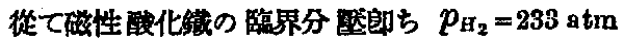
以下に於ては理輪上最も生成し易き $\mathrm{Fe}_{8} \mathrm{O}_{4} \cdot \mathrm{aq}$ 沈

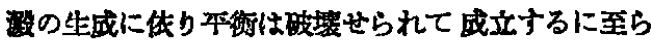

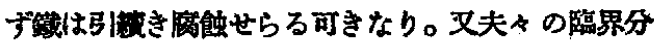
堅以上に於ては不能和狀態にありて，例一は隐界分 竪の最も大なる $\mathrm{F}_{3} \mathrm{O}_{4} \cdot{ }_{9} p^{\mathrm{H}_{2}}=233 \mathrm{~atm}$ の下に溶度 稳の最小なる $\mathrm{Fe}_{3} \mathrm{O}_{4} \cdot \mathrm{aq}$ 飽和溶液と 平衡狀熊にある イオン活度栍は

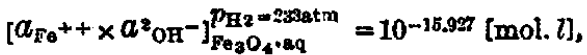

$$
\begin{aligned}
& \left.\left[a_{\mathrm{Fe}}+++\times a^{8} \mathrm{OH}^{-}\right]_{\mathrm{Fe}_{3} \mathrm{O}_{4} .2 \mathrm{Pq}}^{\mathrm{pH}_{2}=28 \mathrm{stm}}=10^{-48.759} \text { [mol. } l\right]
\end{aligned}
$$

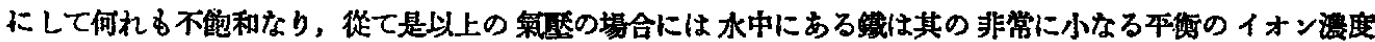

第 2 圆 践の存在せざる水中の平衡

(但し $\mathrm{Fe}_{8} \mathrm{O}_{8} \cdot \mathrm{aq}_{(\mathrm{s})}$ 存在)

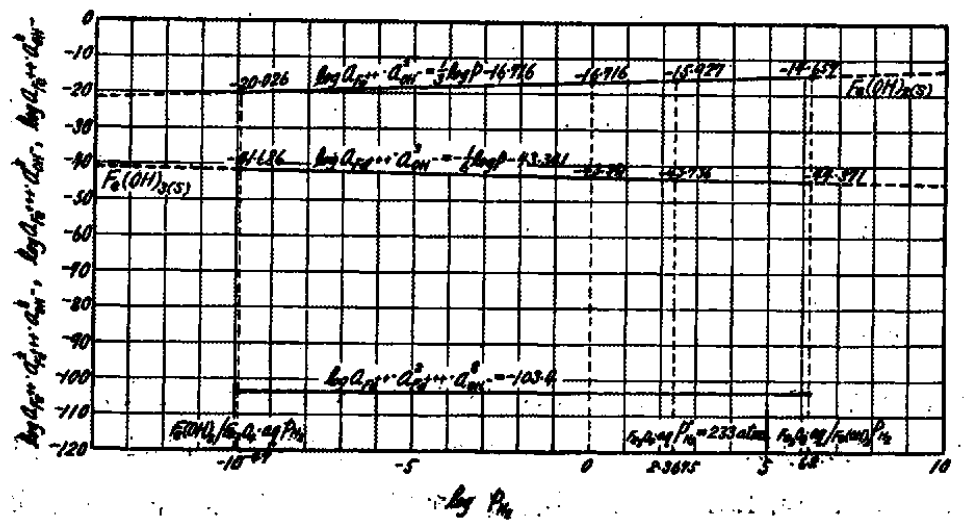

に到䞳すれば病強は停止す可 L。邲方 $p_{\mathrm{E}_{2}}=233 \mathrm{~atm}$ 上

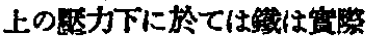
上腐蝕せられざるるのと言ふ を得。又容器の密開せられた る場合に於ては度蝕に体りて

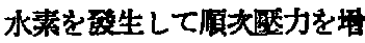
加し蘶に $233 \mathrm{~atm}$ に到達す

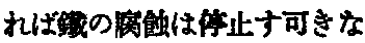
bo

(ii) $\mathrm{Fe}$ の存在せざる堨合 次に金局鐵の存在せざる埥合 
に就て考察するに前の第 2 報 (27) 式に偖り

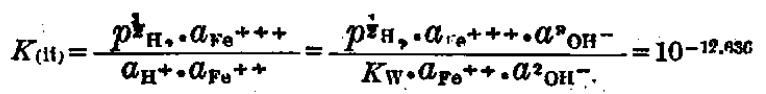

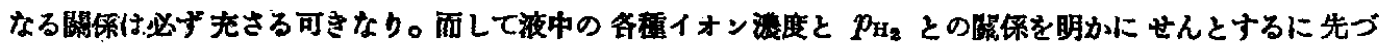

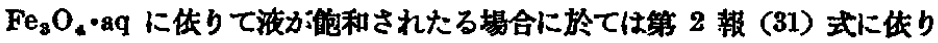

$$
a_{\mathrm{Fn}^{++}}+\times a^{2} \mathrm{rt}^{+++} \times a^{8} \mathrm{OH}^{-}=10^{-10: 4}[\mathrm{~mol} \cdot 1]
$$

なる料限を受く, 從て $\left(27^{\prime}\right)$, (31) より次の兩式を誘薄し得。

$$
\begin{aligned}
& a_{\mathrm{Fu}^{+++}} \times a^{2} \mathrm{OH}^{-}=p^{\frac{1}{3}} \mathrm{H}_{2} \times 10^{-0.718} \\
& a_{\mathrm{Fe}^{+++}} \times a_{\mathrm{OH}^{-}}^{8}=p_{\mathrm{H}^{-\frac{1}{8}}} \times 10^{-48.341}
\end{aligned}
$$

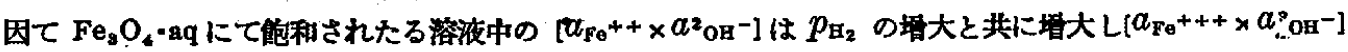

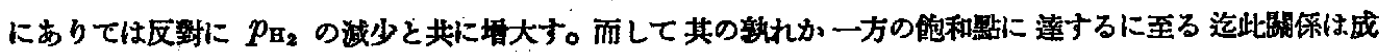

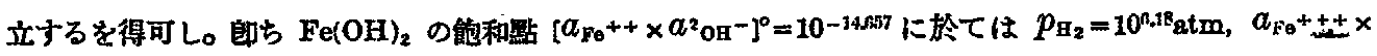
$a^{3} \mathrm{OB}^{-}=10^{-44.871}$ となり $10^{8.18} \mathrm{~atm}$ 以上の $p_{\mathrm{H}_{2}}$ にては固態の $\mathrm{Fe}_{\mathrm{a}} \mathrm{O}_{4} \cdot \mathrm{gq}$ は存在し得ず固熊の $\mathrm{Fe}(\mathrm{OH})_{2} の$

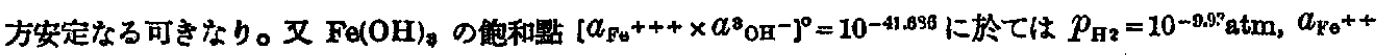
$x a^{2} \mathrm{OH}^{-}=10^{-29.026}$ となり是以下の $p_{\mathrm{H}_{2}}$ にて〕固態の $\mathrm{Fe}_{3} \mathrm{O}_{4} \cdot \mathrm{aq}$ は存在し得ず，此場合は固態の $\mathrm{Fe}(\mathrm{OH})_{8}$

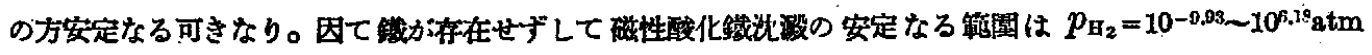

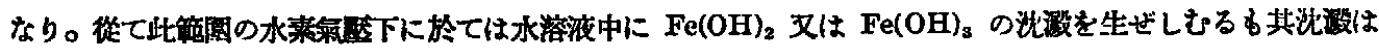
理諭上不安定にして

$$
\begin{aligned}
& 3 \mathrm{Fe}(\mathrm{OH})_{2}=\mathrm{Fe}_{8} \mathrm{O}_{4}+\mathrm{H}_{2}+2 \mathrm{H}_{2} \mathrm{O} \\
& 3 \mathrm{Fe}(\mathrm{OH})_{8}+\frac{1}{2} \mathrm{H}_{2}=\mathrm{Fe}_{2} \mathrm{O}_{4}+5 \mathrm{H}_{2} \mathrm{O}
\end{aligned}
$$

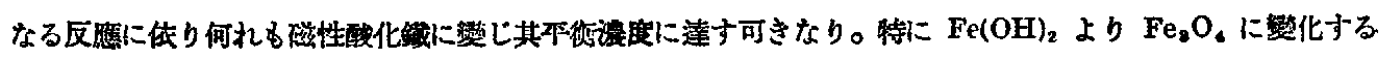

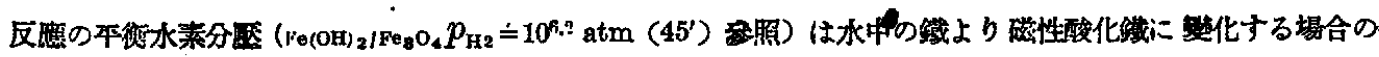
$p_{\mathrm{H}_{2}}=10^{\circ}$ 湔, $233 \mathrm{~atm}$ に比し非常に大にして著しく容易に磁性酸化鐵に薙化する倾向むるを示するのなり。而

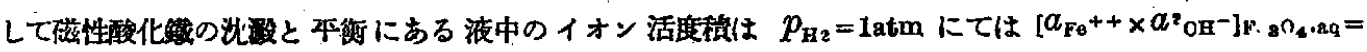

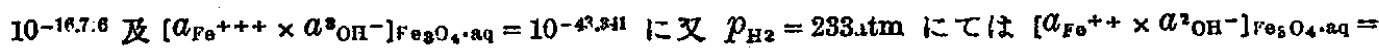

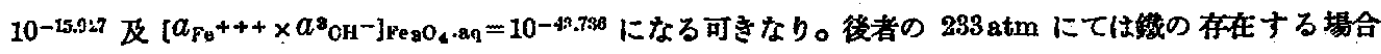

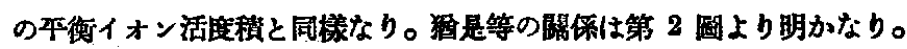

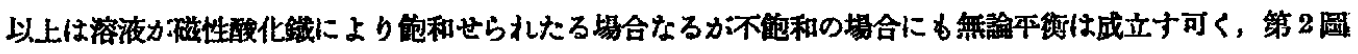

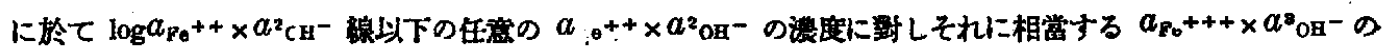
盟力: $\log a_{\mathrm{re}}{ }^{+++.} a^{2} \mathrm{CH}^{-}$の楾の下に存在す可きなり。

$$
\text { 遊離エネルギーの計算 }
$$

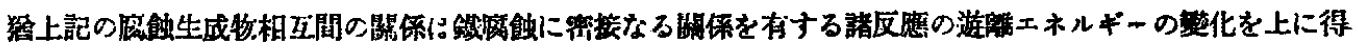

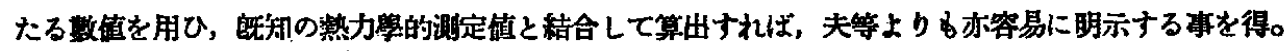

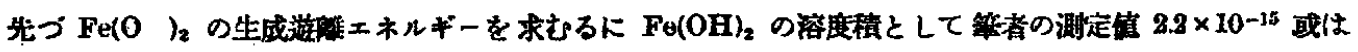

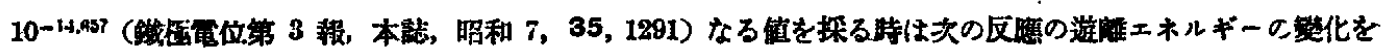


稩算する事を得。

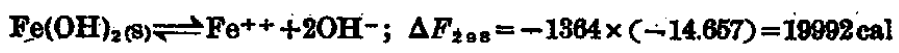

ヌルウィス氏知算值 (Lewis \& Randali, “Thermodynamios", 1823) に估り

$$
\frac{1}{2} \mathrm{O}_{2}+\frac{1}{2} \mathrm{H}_{2}+\Theta=\mathrm{OH}^{-} ; \Delta F_{2 \mathrm{~B}}=-37455 \mathrm{cals}
$$

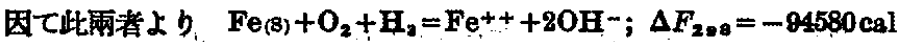

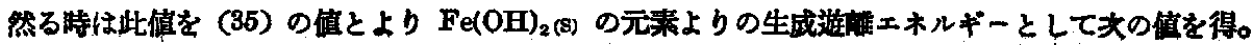

$$
\mathrm{Fe}(\mathrm{s})+\mathrm{O}_{2}+\mathrm{H}_{2}=\mathrm{Fe}(\mathrm{OH})_{2}(\mathrm{~s}) ; \quad \Delta F_{28.8}=-114572 \mathrm{cal}
$$

1) 水中の践より $\mathrm{Fe}(\mathrm{OH})_{2}$ の生成遊離エネルギー, 前第 1 報 (9) の $\mathrm{Fe}(\mathrm{OH})_{2} p^{\circ} \mathrm{H}_{2}=12.53 \mathrm{~atm}$ なる值を 用ひて直ちに計算する事を得。

$$
\begin{aligned}
\mathrm{Fe}_{(\mathrm{B})}+2 \mathrm{H}_{2} \mathrm{O}_{(1)} \rightleftharpoons \mathrm{Fe}(\mathrm{OH})_{2}(\mathrm{~S})+\mathrm{H}_{2} ; & \\
\Delta F_{288} & =-R T \ln _{\mathrm{Fe}(\mathrm{OH})_{2}} P_{\mathrm{H}_{2}}^{\circ}=-1364 \log 12.53=-1498 \mathrm{csl}
\end{aligned}
$$

從て任意の $p_{\mathrm{H}_{2}}$ の水素を生ずるるのとすれば

$$
\mathrm{Fe}(\mathrm{s})+2 \mathrm{H}_{2} \mathrm{O}_{(\mathrm{l})} \rightleftharpoons \mathrm{Fe}(\mathrm{OH})_{2}(\mathrm{~s})+\mathrm{H}_{2}\left(p_{\mathrm{H}_{2}}\right) ; \quad \Delta F_{288}=-1498+1364 \log p_{\mathrm{B}_{2} \text { cal }}
$$

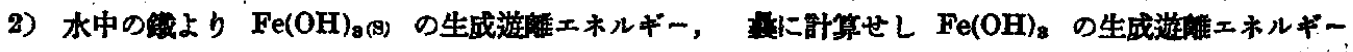

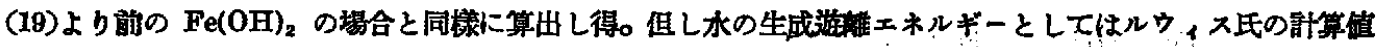
-56560cal (Lewis \& Randall, “Thermodynamics”,) あれども，篗者は本研究の計算に當り $K_{W}=10^{-13.98}$ (I.C.T. VI, 152) を正しきるのとして $\frac{1}{2} \mathrm{O}_{2}+\frac{1}{2} \mathrm{H}_{2}+\Theta=\mathrm{OH}-; \Delta F_{208}=-37455 \mathrm{cal}$ を探用せしを以て其 結果水の生成遊触エネルギーとして算出せらるる夫の値を探用す。 (36) と (37) とより

$$
\mathrm{H}_{2}+\frac{1}{2} \mathrm{O}_{2}=\mathrm{H}_{2} \mathrm{O}_{(1)} ; \Delta F_{208}=\frac{-114572-(-1498)}{2}=-56537 \mathrm{cal}
$$

因に此值は $-56540 \mathrm{cal}$ (Lewis \& Randall, "Thermodynamics," 1923, p. 484), -56520 cal (Eastman, Bur. Mines, Büll., Circ. 6125 (1929)) 及 -56553 cal (Benton \& Drake, J. Amer. Chem. Soc̀., 1932, 54, 2186）と良く一政せるるのなり。

$$
\begin{aligned}
& \mathrm{Fe}(\mathrm{s})+3 \mathrm{H}_{2} \mathrm{O}_{(\mathrm{l})}=\mathrm{Fe}(\mathrm{OH})_{8}(\mathrm{~B})+1 \frac{1}{2} \mathrm{H}_{2}(p) ; \\
& \Delta F_{2 \mathrm{~B}}=-171660+1364 \log p_{\mathrm{H}_{2}}^{\mathrm{g}}-3(-56537) \mathrm{cal}
\end{aligned}
$$

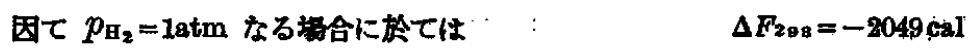

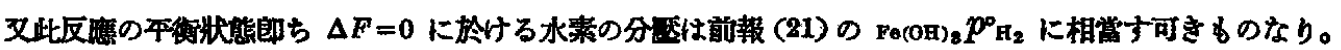

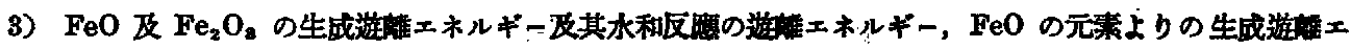
ネルギーとして Ralston 氏及 Halferdahl 氏仕各々-57472 cal (Ralston, Bur. Mines, Bull., 1929, 296 及 -58140 cal (Eastman, Bur. Mines, Bull., Circ. 1929, 6125; Halferdahl, J. Ind. Eng. Chem., 1930, 22,

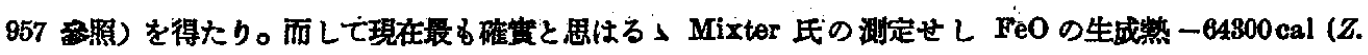

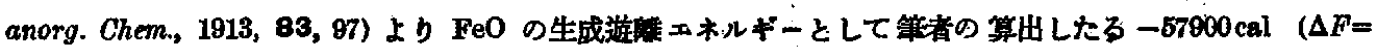
$\Delta H-T \Delta S,-64300+288.1 \times(9.41$ (Ralston) -6.87 (Ralston)-24)=-57899 cal; Ralston, Bur. Mines, Bull.,

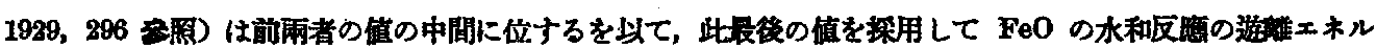
ギーを部幕すれば夫の如し。 


$$
\mathrm{FeO}_{(\mathrm{B})}+\mathrm{H}_{2} \mathrm{O}_{(1)}=\mathrm{Fe}(\mathrm{OH})_{2(\mathrm{~B})} ; \Delta F_{2 \mathrm{Oa}}=-114572-(-57900-56537)=-135 \mathrm{cal} \text { (40) }
$$

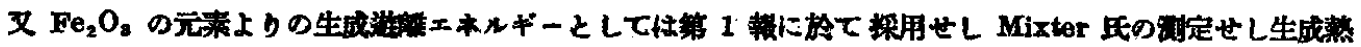
$-192200 \mathrm{cal}$ (Mixter, $Z$. anorg. Chem., 1913, 83, 97) よ b算出せる Ralston 氏の舒蕉值 $\Delta F_{200}=-173050$

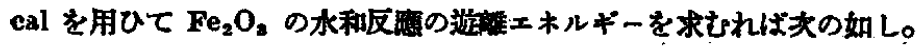

$$
\begin{aligned}
\mathrm{Fe}_{2} \mathrm{O}_{8(\mathrm{~B})}+3 \mathrm{H}_{2} \mathrm{O}_{(\mathrm{B})} & =2 \mathrm{Fe}(\mathrm{OH})_{8(\mathrm{~B})} ; \\
\Delta F_{28 \mathrm{~s}} & =2(-171660)-(-173050+3 \times-56537)=-659 \mathrm{cal}
\end{aligned}
$$

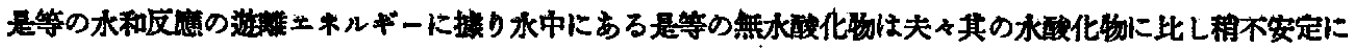

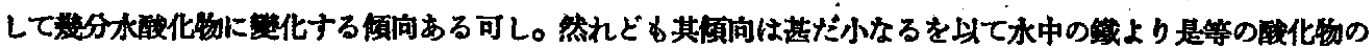

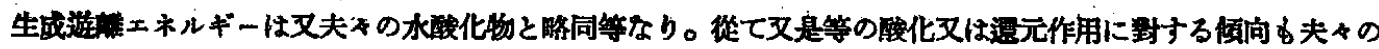
水酸化物と同程度なりと見䰻して差支なかる可し。

$$
\begin{array}{r}
\mathrm{Fe}(\mathrm{g})+\mathrm{H}_{2} \mathrm{O}_{(\mathrm{l})}=\mathrm{FeO}_{(\mathrm{s})}+\mathrm{H}_{2} ; \Delta F_{298}=-57900-(-56537)=-1363 \mathrm{cal} \\
2 \mathrm{Fe}(\mathrm{g})+3 \mathrm{H}_{2} \mathrm{O}_{(\mathrm{l})}=\mathrm{Fe}_{2} \mathrm{O}_{2(\mathrm{~s})}+3 \mathrm{H}_{2} ; \\
\Delta F_{298}=-173050-3(-56537)=-3439 \mathrm{cal}
\end{array}
$$

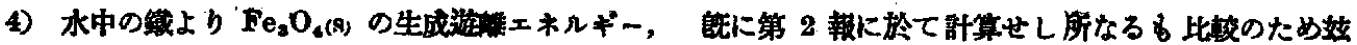
に再錄すれば

$$
\begin{aligned}
& 3 \mathrm{Fe}_{(\mathrm{S})}+4 \mathrm{H}_{2} \mathrm{O}_{(\mathrm{s})}=\mathrm{Fe}_{2} \mathrm{O}_{4}(\mathrm{~s})+4 \mathrm{H}_{2} ; \\
& \Delta F_{2 \mathrm{es}}=-239065-4(-56537)=-12917 \mathrm{cal} .
\end{aligned}
$$

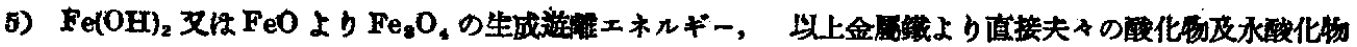

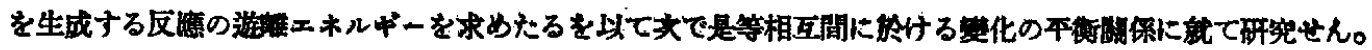
$3 \mathrm{Fe}(\mathrm{OH})_{2}(\mathrm{~s})=\mathrm{Fe}_{2} \mathrm{O}_{4}(\mathrm{~s})+2 \mathrm{H}_{2} \mathrm{O}(\mathrm{l})+\mathrm{H}_{2}(p)$;

$$
\Delta F_{2 \mathrm{es}}=-238065^{\circ}+2(-56537)+1364 \log p_{\mathrm{H}_{2}}-3(-114572)
$$

$p_{\mathrm{H}_{2}=1 \mathrm{~atm}: \Delta F_{2 \mathrm{~g}}=-8515 \mathrm{cal}}$

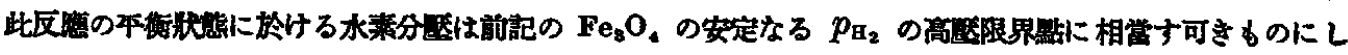
て此式に $\Delta F=0$ を代入して直ちに其出子るるを得。郎ち

$$
\Delta F=0: \quad \mathrm{Fe}(\mathrm{OH})_{2} / \mathrm{Feg}_{2} p_{\mathrm{H}_{2}}=10^{\mathrm{a}, 2} \mathrm{~atm}
$$

又 $\mathrm{FeO}$ に就ては $3 \mathrm{FeO}_{(\mathrm{B})}+\mathrm{H}_{2} \mathrm{O}_{())}=\mathrm{Fe}_{3} \mathrm{O}_{4}(\mathrm{~A})+\mathrm{H}_{2}(P)$;

$$
\begin{aligned}
& \Delta F_{2 B B}=-239065+1364 \log p_{\mathrm{H} 2}-3(-57900)-(-56597) \\
& p_{\mathrm{Bz}}=1 \text { atm: } \quad \Delta F_{20 \mathrm{a}}=-8828 \mathrm{cal}
\end{aligned}
$$

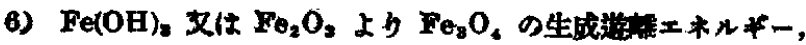

$$
\begin{aligned}
& 3 \mathrm{Fe}(\mathrm{OH})_{8}(\mathrm{~s})+\frac{1}{2} \mathrm{H}_{2}=\mathrm{Fe}_{2} \mathrm{O}_{4(8)}+5 \mathrm{H}_{2} \mathrm{O}_{(1)} \text {; } \\
& \Delta F_{298}=-239065+5(-56587)-3(-271660)=-6770 \mathrm{cal} \\
& \frac{3}{2} \mathrm{Fe}_{2} \mathrm{O}_{2(\mathrm{~s})}+\frac{1}{2} \mathrm{H}_{2}=\mathrm{Fe}_{2} \mathrm{O}_{4(3)}+\frac{1}{2} \mathrm{H}_{2} \mathrm{O}_{(1)} ; \\
& \Delta F_{\text {Q }}=-239065+\frac{1}{2}(-56537)-\frac{3}{2}(-173050)=-7759 \mathrm{cal}
\end{aligned}
$$

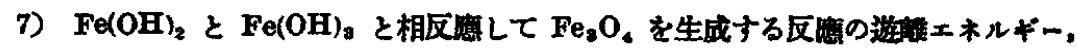


$\mathrm{Fe}(\mathrm{OH})_{2}(\mathrm{~A})+2 \mathrm{Fe}(\mathrm{OH})_{2(\mathrm{~s})}=\mathrm{Fe}_{2} \mathrm{O}_{4}(\mathrm{\theta})+4 \mathrm{H}_{2} \mathrm{O}_{(\alpha)}$;

$$
\Delta F_{290}=-239005+4(-56587)-(-114572+2 x-171660)=-7821 \mathrm{cal}
$$

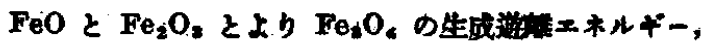

$$
\begin{aligned}
& \mathrm{FeO}_{(8)}+\mathrm{Fe}_{2} \mathrm{O}_{4}(\mathrm{~g})=\mathrm{Fe}_{8} \mathrm{O}_{4}(\mathrm{~B}) ; \\
& \Delta F_{208}=-239065-(-57900-173050)=-8116 \mathrm{cal} \\
& \text { 水莱氣中に於ける腐蝕機䅘 }
\end{aligned}
$$

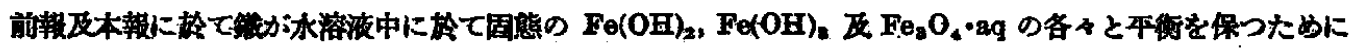

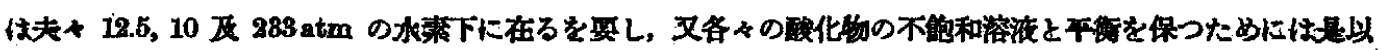

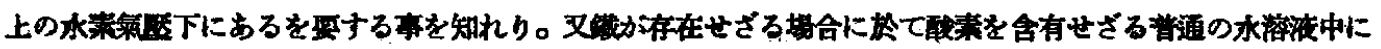

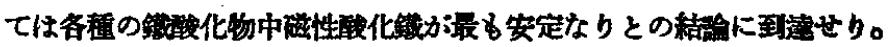

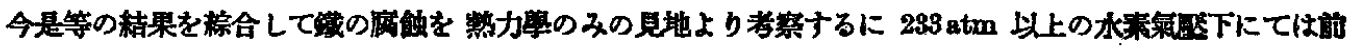

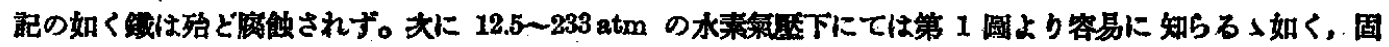

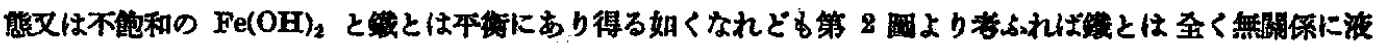

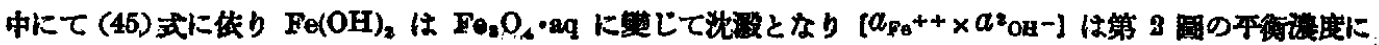

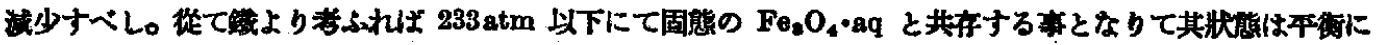

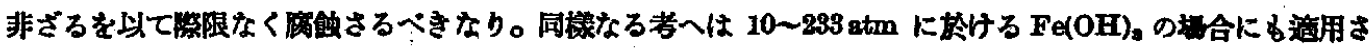

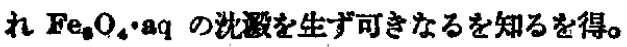

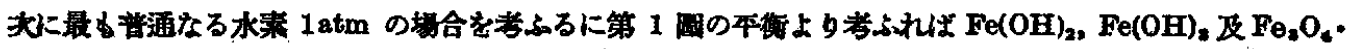

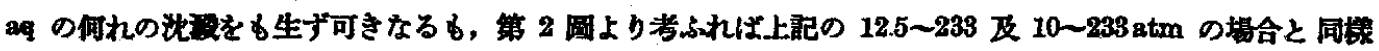

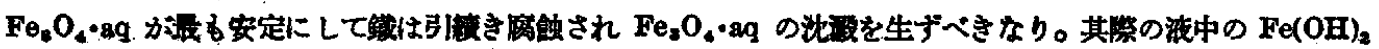

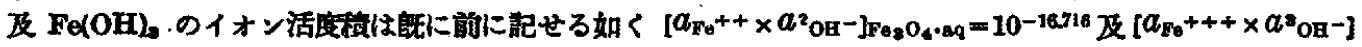

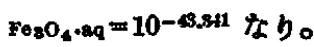

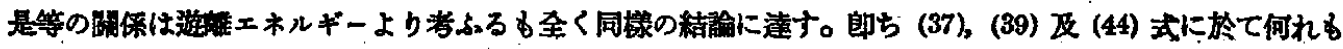

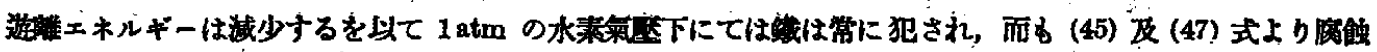
生成物は $\mathrm{Fe}(\mathrm{OH})_{2}$ 及は $\mathrm{Fe}(\mathrm{OH})_{3}$ ならずして $\mathrm{Fe}_{3} \mathrm{O}$ ×・aq なる可きを知るを得。

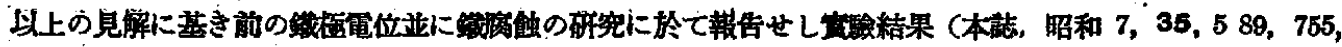
1291)を考案せんとす。

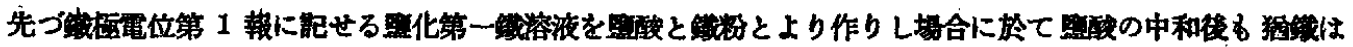

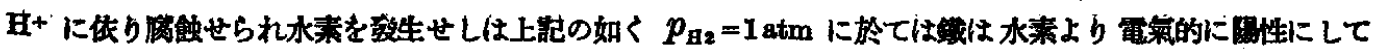

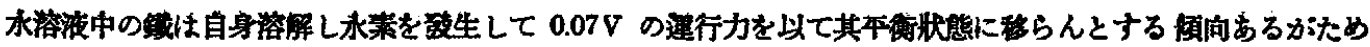

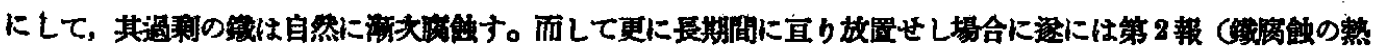

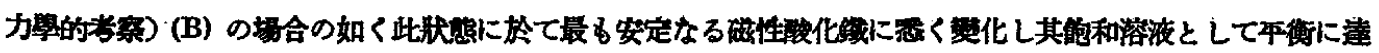

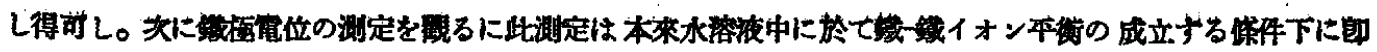
ち

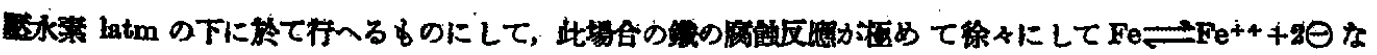

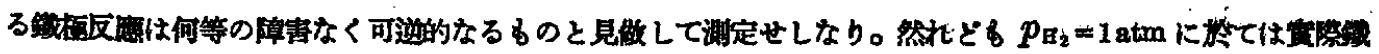




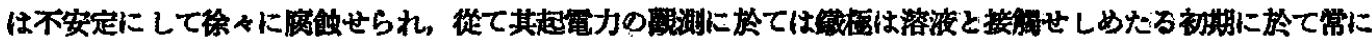

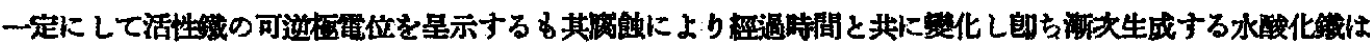

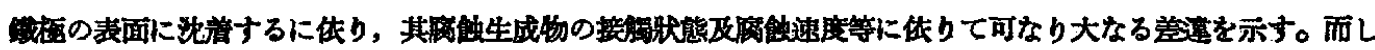

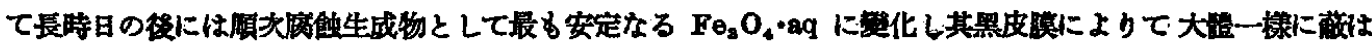

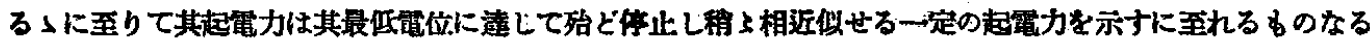
可L。

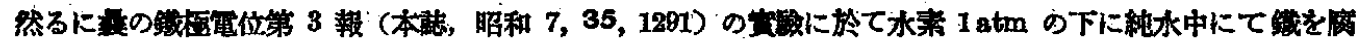

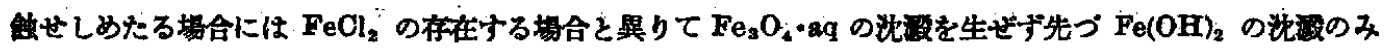

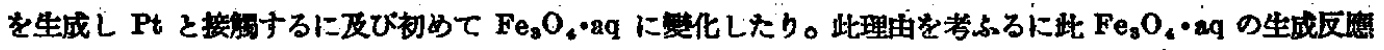

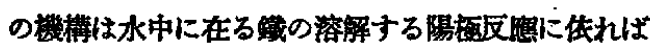
a) $\mathrm{Fe} \rightarrow \mathrm{Fe}^{++}+2 \Theta$,
$E^{\circ} \mathrm{Fi}_{\mathrm{O}} \rightleftharpoons \mathrm{Fu}^{++}=-0.4285 \mathrm{~V}$
b) $\mathrm{Fe} \longrightarrow \mathrm{Fe}^{++++3 \Theta}$,
$\mathrm{E}^{\circ} \mathrm{F \theta} \rightleftharpoons \mathrm{F \theta}+++=-0.0352 \mathrm{~V}$
c) $\mathrm{Fe}^{++} \longrightarrow \mathrm{Fe}^{+++}+\Theta$,
$\mathrm{E}^{\circ} \mathrm{Fe}^{++} \rightleftharpoons \mathrm{Fe}^{+++}=+0.7473 \mathrm{~V}$

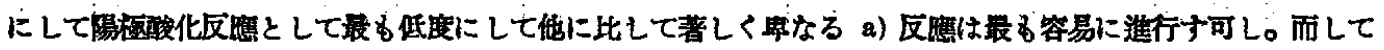

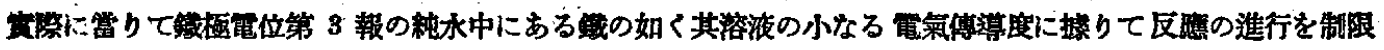

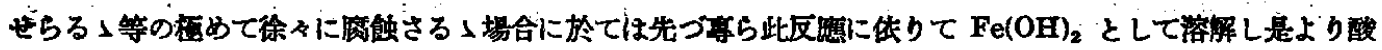

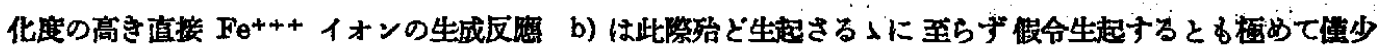

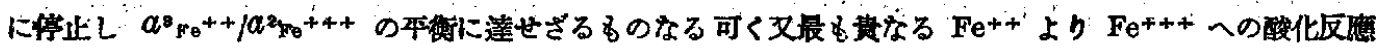

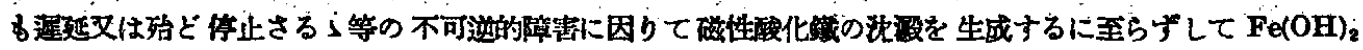

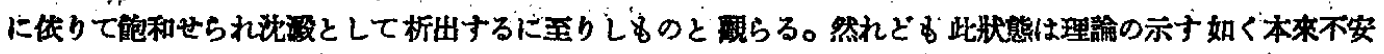
定なるものにして Pt ヌは国類等の存在する 是等反潐の進行に適當なる 狀態に於て更に進展して最る安定な る $\mathrm{Fe}_{3} \mathrm{O}$ ・・aq を沈淑するに至るるのなる可し。

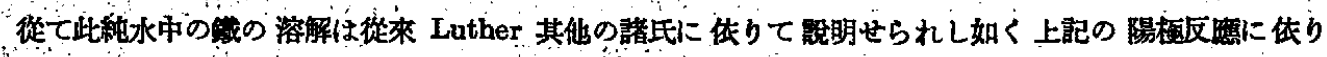

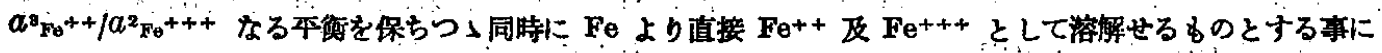

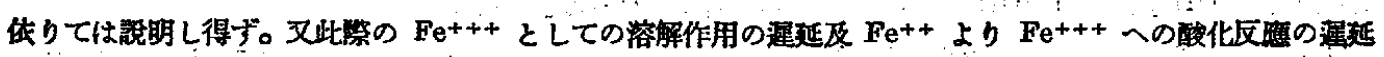

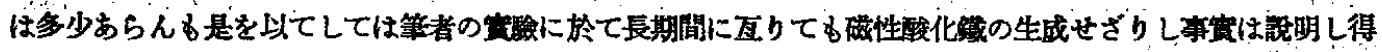

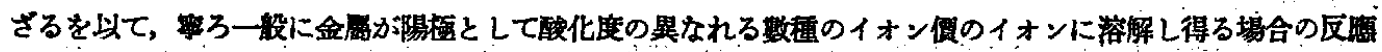

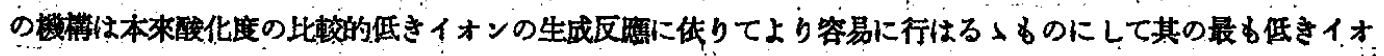

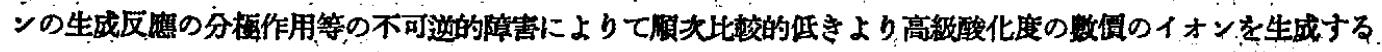

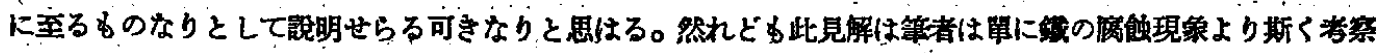
せるすのにして般には多数の研究に侯ちて確定さる可きなり。

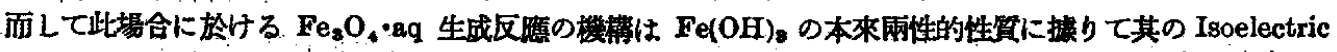

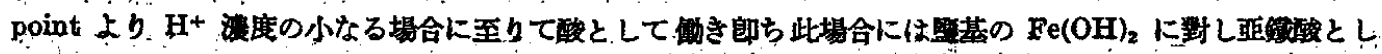

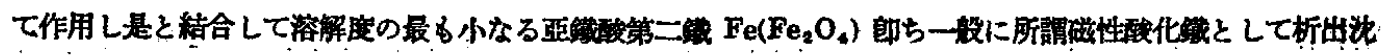
設なるに琹れるすのと思惟せらる。 
緿

括

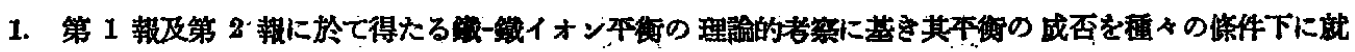

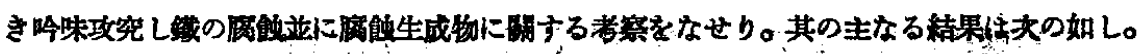

\section{$p_{\mathrm{H}_{2}}$ (i) Fe の共存する場合}

$200 \mathrm{~atm} \mathrm{Fe}_{3} \mathrm{O}_{4} \cdot \mathrm{aq}$ 鴒和, 平嘼成立, 府触传止

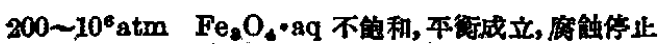

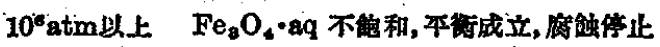

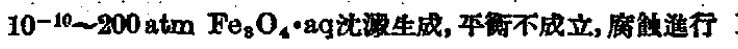

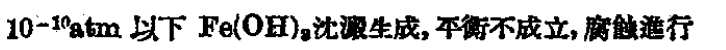

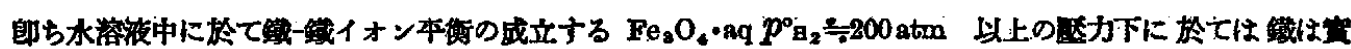

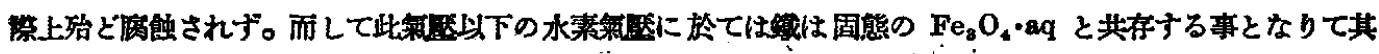

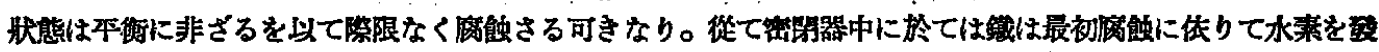

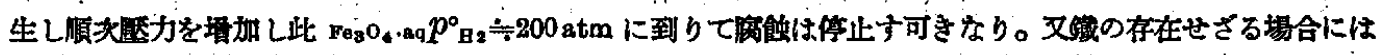

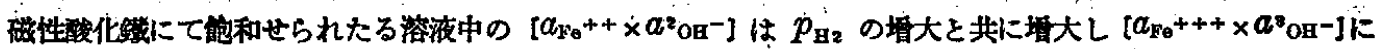

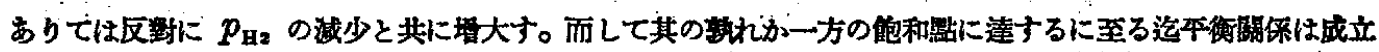

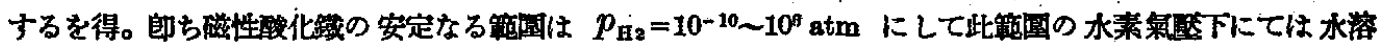

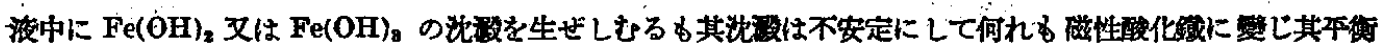

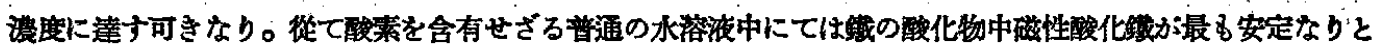
云与事を得可し。唒是等の關你は游離エネルギー上り考ふるるる全く同樣の結論に到達す。

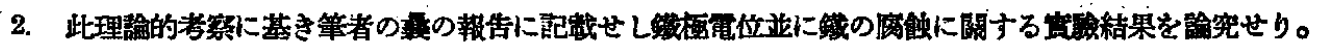

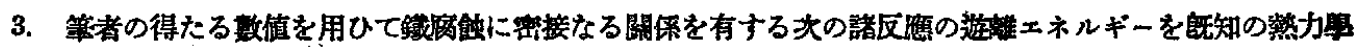
的測定值と結合して算出せり。

$$
\begin{aligned}
& \mathrm{Fe}(\mathrm{OH})_{2}(\mathrm{~s}) \rightleftharpoons \mathrm{Fe}^{+++2 \mathrm{OH}-;} \\
& \mathrm{Fe}(\mathrm{S})+\mathrm{O}_{2}+\mathrm{H}_{2}=\mathrm{Fe}(\mathrm{OHI})_{2}(\mathrm{~B}) \text {; } \\
& \mathrm{Fe}(\mathrm{s})+2 \mathrm{H}_{2} \mathrm{O}_{(\mathrm{l})}=\mathrm{Fe}(\mathrm{OH})_{2}(\mathrm{~s})+\mathrm{H}_{2} \text {; } \\
& \mathrm{Fe}_{(\mathrm{B})} 3 \mathrm{H}_{2} \mathrm{O}_{(\mathrm{l})}=\mathrm{Fe}(\mathrm{OH})_{3}(\mathrm{~s})+1 \frac{l}{2} \mathrm{H}_{2} \text {; } \\
& \mathrm{FeO}_{(\mathrm{S})}+\mathrm{H}_{2} \mathrm{O}_{(\mathrm{l})}=\mathrm{Fe}(\mathrm{OH})_{2(\mathrm{~s})} \text {; } \\
& \mathrm{Fe}_{2} \mathrm{O}_{3}(\mathrm{~s})+3 \mathrm{H}_{2} \mathrm{O}_{(j)}=2 \mathrm{Fe}(\mathrm{OH})_{3}(\mathrm{~s}) ; \\
& \mathrm{Fe}(\mathrm{s})+\mathrm{H}_{2} \mathrm{O}_{(\mathrm{l})}=\mathrm{FeO}(\mathrm{s})+\mathrm{H}_{2} \text {; } \\
& 2 \mathrm{Fe}(\mathrm{s})+3 \mathrm{H}_{2} \mathrm{O}_{(1)}=\mathrm{Fe}_{2} \mathrm{O}_{3}(\mathrm{~s})+3 \mathrm{H}_{2} \text {; } \\
& 3 \mathrm{Fe}(\mathrm{S})+4 \mathrm{H}_{2} \mathrm{O}_{()}=\mathrm{Fe}_{3} \mathrm{O}_{4}(\mathrm{~S})+4 \mathrm{H}_{2} \text {; } \\
& 3 \mathrm{Fe}(\mathrm{OH})_{2}(\mathrm{~s})=\mathrm{Fe}_{2} \mathrm{O}_{6}(\mathrm{~s})+2 \mathrm{H}_{2} \mathrm{O}_{(\mathrm{l})}+\mathrm{H}_{2} \text {; } \\
& 3 \mathrm{FeO}_{(\mathrm{S})}+\mathrm{H}_{2} \mathrm{O}_{(\mathrm{l})}=\mathrm{Fe}_{8} \mathrm{O}_{4}(\mathrm{~s})+\mathrm{H}_{2} \text {; } \\
& 3 \mathrm{Fe}(\mathrm{OH})_{8(\mathrm{~S})}+\frac{1}{2} \mathrm{H}_{2}=\mathrm{Fe}_{2} \mathrm{O}_{4}(\mathrm{~s})+5 \mathrm{H}_{2} \mathrm{O}_{(\mathrm{l})} \text {; } \\
& \frac{3}{2} \mathrm{Fe}_{2} \mathrm{O}_{3}(\mathrm{~s})+\frac{1}{2} \mathrm{H}_{2}=\mathrm{Fe}_{2} \mathrm{O}_{4}(\mathrm{~s})+\frac{1}{2} \mathrm{H}_{2} \mathrm{O}_{(\mathrm{D})} \text {; } \\
& \mathrm{Fe}(\mathrm{OH})_{2(\mathrm{~s})}+2 \mathrm{Fe}(\mathrm{OH})_{8(\mathrm{~s})}=\mathrm{Fe}_{3} \mathrm{O}_{4}(\mathrm{~S})+4 \mathrm{H}_{2} \mathrm{O}_{(1)} \text {; } \\
& \mathrm{FeO}_{(\mathrm{s})}+\mathrm{Fe}_{2} \mathrm{O}_{8}(\mathrm{~s})=\mathrm{Fe}_{2} \mathrm{O}_{4}(8) ; \\
& \Delta F_{29 \mathrm{a}}=19992 \mathrm{cal} \\
& \Delta F_{298}=-114572 \mathrm{cal} \\
& \Delta F_{288}=-1498 \text { cal } \\
& \Delta F_{2 \mathrm{ag}}=-2049 \mathrm{cal} \\
& \Delta F_{29 \mathrm{a}}=-135 \mathrm{cal} \\
& \Delta F_{298}=-659 \text { cal } \\
& \Delta F_{298}=-1363 \text { cal } \\
& \Delta F_{298}=-3439 \text { cal } \\
& \Delta F_{298}=-12917 \mathrm{cal} \\
& \Delta F_{298}=-8515 \mathrm{cal} \\
& \Delta F_{298}=-8828 \mathrm{cal} \\
& \Delta F_{288}=-6770 \text { cal } \\
& \Delta F_{298}=-7759 \text { cal } \\
& \Delta F_{298}=-7321 \mathrm{cal} \\
& \Delta F_{28 \mathrm{~B}}=-8115 \mathrm{cal}
\end{aligned}
$$

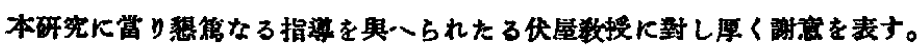

